\title{
Comparison of Immunohistochemistry Assay Results with Gene Expression Profiling Methods for Diffuse Large B-Cell Lymphoma Subtype Identification in Matched Patient Samples
}

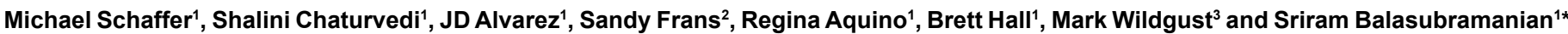
${ }^{1}$ Janssen Oncology Translational Research, Spring House, PA, USA

2Janssen Research and Development, Beerse, Belgium

${ }^{3}$ Janssen Research and Development, Raritan, NJ, USA

\begin{abstract}
Background: The cell of origin (COO) in diffuse large B-cell lymphoma (DLBCL) has prognostic importance. While the $\mathrm{COO}$ was originally classified into germinal center B-cell (GCB) or activated B-cell (ABC) subtypes by microarray analysis, routine use was not practical. Immunohistochemistry (IHC)-based methods are widely used with varying results due to lack of standardization. Several classification methods have been developed recently; understanding the concordance between these and existing methods is essential to their practical application. Therefore, we evaluated concordance between 3 commercial assays: a standardized Hans-based IHC method and 2 gene expression profiling (GEP) methods and compared these to the accepted microarray classification.
\end{abstract}

Methods: 137 DLBCL-confirmed tumor samples were evaluated using a standardized Hans-based IHC method for GCB or non-GCB subtype, by a published microarray-based assay, a digital gene expression-based Lymphoma Subtyping Test (LST) and a next-generation sequencing-based assay (EdgeSeq COO). Subtype calls from the 3 GEP methods were harmonized to "GCB" or "non-GCB" and assessed for concordance.

Results: Concordance between the Hans-based IHC assay and the microarray-based assay, LST assay and EdgeSeq assay was $79.6 \%(\mathrm{~N}=137), 80.0 \%(\mathrm{~N}=125)$ and $78.2 \%(\mathrm{~N}=64)$, respectively; the positive percent agreement (PPA) in non-GCB was $88.9 \%, 87.0 \%$ and $78.1 \%$, respectively. Concordance for the Hans-based IHC assay versus GEP methods was especially high for direct GCB calls $(91.0 \%, 88.3 \%$ and $78.1 \%$ for microarray, LST and EdgeSeq $\mathrm{COO}$ methods, respectively). The newly developed GEP assays performed well against the microarray GEP method, against which they were calibrated (concordance $93.7 \%$ and $87.5 \%$ and PPA $94.3 \%$ and $92.9 \%$, respectively, for LST and EdgeSeq COO).

Conclusion: These results demonstrated good consistency between various platforms for stratification of DLBCL into COO subtype classifications. Application of a standardized Hans-based IHC assay offers a robust, rapid and easily accessible platform to classify DLBCL into prognostically important subtypes.

Keywords: Diffuse large B-cell lymphoma; Hans-based immunohistochemistry; Gene expression profiling

\section{Introduction}

Diffuse large B-cell lymphoma (DLBCL) is the most common type of lymphoma, accounting for $30 \%$ to $35 \%$ of non-Hodgkin lymphoma $[1,2]$. DLBCL is a heterogeneous disease and has distinct molecular subtypes based on the cell of origin (COO). Methods such as immunohistochemistry (IHC) and gene expression profiling (GEP) are among the more common approaches used to classify subtypes of DLBCL. GEP using DNA microarrays has been the standard experimental approach to molecularly classify DLBCL into major COO subtypes: germinal center B-cell-like (GCB), activated B-cell-like (ABC) and primary mediastinal B-cell lymphoma (PMBL) [3-6]. The small number of patients who cannot be classified clearly into these molecular subtypes are referred to as "unclassified" (UNC).

Depending on the molecular subtype, the pathobiology of the disease is diverse; other than PBML, the initial clinical presentation of each subtype is similar but associated with very different clinical outcomes and large underlying molecular diversity. Of these biological factors, the $\mathrm{COO}$ has proved to be the most important, and it is now accepted that COO is a prognostic factor in DLBCL [7-9].

DLBCL subtypes respond to therapy in very different ways [10]. For example, patients with ABC DLBCL who failed R-CHOP (rituximab, cyclophosphamide, doxorubicin, vincristine and prednisone) therapy have shown favorable responses to the Bruton's tyrosine kinase (BTK) inhibitor ibrutinib [11] or the immunomodulatory drug lenalidomide [12], alone or in combination with chemotherapy. This provides a rationale for the selective development of treatments for subtypes of DLBCL classified by COO, which further emphasizes the need for rapid and accurate identification of these subtypes.

Most patients with PMBL have a favorable outcome with a 3-year overall survival rate of $97 \%$ [2]. In clinical practice today, PMBL is easily diagnosed by other methods; thus, DLBCL COO classification efforts have focused on distinguishing the remaining subtypes.

The accepted standard for classification of DLBCL subtypes has

*Corresponding author: Sriram Balasubramanian, Janssen Oncology Translational Research, 1400 McKean Road, Spring House, PA 19087, USA, Tel: +1 2157937757; E-mail: sbalas14@its.jnj.com

Received March 03, 2018; Accepted March 21, 2018; Published March 24, 2018

Citation: Schaffer M, Chaturvedi S, Alvarez JD, Frans S, Aquino R, et al. (2018) Comparison of Immunohistochemistry Assay Results with Gene Expression Profiling Methods for Diffuse Large B-Cell Lymphoma Subtype Identification in Matched Patient Samples. J Mol Biomark Diagn 9: 386. doi: 10.4172/21559929.1000386

Copyright: @ 2018 Schaffer M, et al. This is an open-access article distributed under the terms of the Creative Commons Attribution License, which permits unrestricted use, distribution, and reproduction in any medium, provided the original author and source are credited. 
Citation: Schaffer M, Chaturvedi S, Alvarez JD, Frans S, Aquino R, et al. (2018) Comparison of Immunohistochemistry Assay Results with Gene Expression Profiling Methods for Diffuse Large B-Cell Lymphoma Subtype Identification in Matched Patient Samples. J Mol Biomark Diagn 9: 386. doi: 10.4172/2155-9929.1000386

been using microarray GEP [13]. However, a reproducible, easy-to-use and validated microarray-based GEP assay for DLBCL subtyping has not been accessible to all medical communities and any microarray GEP-based approach generally has a turnaround time of 2 to 3 weeks, which makes it difficult to implement as a prospective selection marker. Therefore, routine clinical practice in DLBCL has used standard IHCbased methods, which are widely available in most clinical settings, to classify patients for therapy. Much effort has been dedicated to developing robust IHC assays using various combinations of CD10, BCL6, BCL2, MUM1/IRF4, FOXP1, GCET1 and LMO2 proteins, all of which have been identified as having differential expression in the DLBCL subtypes. These markers have been used to develop at least 9 different IHC tests [14]. The Hans method was developed in 2004, based on 3 markers: CD10, BCL6 and MUM1/IRF4 [15], and has remained the most popular due to its simplicity with only 3 widely used markers, a consistent $30 \%$ cutoff for all 3 markers and demonstrated prognostic value. This binary algorithm segregates patients into 2 groups - GCB (in $42 \%$ of patients) and non-GCB (in $58 \%$ of patients) - instead of the continuum from which discrete classes are derived from populationbased numeric cutoffs [15]. The non-GCB group incorporates the ABC subtype along with the unclassified group, and as with ABC DLBCL, these non-GCB patients have a less favorable outcome with standard immunochemotherapy [16]. Algorithms for IHC have $80 \%$ to $87 \%$ concordance with GEP and have gained the most clinical acceptance [17-19]. However, there can be limitations to using IHC algorithms, including reproducibility concerns and variability between laboratories; also, the various IHC methods have not been uniformly reported to have the same prognostic utility as GEP [20,21]. A standardized Hans-based IHC kit has recently been developed to overcome these limitations and is currently in clinical development as a companion diagnostic in a large randomized phase III trial evaluating the activity of ibrutinib in combination with R-CHOP versus R-CHOP alone in non-GCB DLBCL patients (NCT01855750) [22].

It is critical to understand the degree of concordance between IHC and GEP methods in classifying DLBCL subtypes, particularly as other gene expression-based methods that attempt to reproduce the microarray-based classification have been developed. Some assays are commercially available, including the Lymphoma Subtyping Test (LST) assay (NanoString, Seattle, WA, USA) and the next-generation sequencing (NGS) EdgeSeq COO assay (HTG Molecular, Tucson, AZ, USA). The LST assay is a commercially available assay in which 20 digital color-coded gene probes hybridize directly to specific nucleic acid targets, thereby allowing detection and quantification of many RNA transcripts in a single reaction [23]; it uses formalinfixed, paraffin-embedded (FFPE) diagnostic blocks and was validated using matched frozen tissue $[23,24]$. This assay is currently under development as a companion diagnostic to enroll subjects in a study evaluating R-CHOP \pm lenalidomide in ABC DLBCL patients [25]. The EdgeSeq $\mathrm{COO}$ assay is another commercially available GEP-based assay to quantitate gene expression and infer a DLBCL subtype [26]. This method has its own proprietary chemistry, utilizes very little sample and produces classification results matching microarraybased GEP. However, this is a novel technology, and it is important to evaluate the performance against the other accepted technologies in the field. These GEP methods have their own limitations in that they are not yet widely available worldwide, require unique and expensive instrumentation, combine RNA from nonhomogeneous samples with widely varying tumor cell percentages, and it can be difficult to obtain sufficient high-quality RNA from FFPE diagnostic blocks or slides for all patients [24]. In contrast, IHC is more amenable for use with FFPE samples and allows more subtle evaluation of the sample and staining characteristics by a pathologist.

Each methodology described has its own limitations; however, optimized assays using some of these methodologies are becoming commercially available, and thus multiple assays may be used to identify DLBCL patients and select the optimum therapy in the clinic. Therefore, it becomes important to compare multiple platforms and evaluate the concordance between these IHC and GEP assays, as well as the concordance across the different GEP assays. To this end, we compared the Hans-based IHC assay with the traditional microarray GEP-based method and the novel gene expression-based assays described above under standard conditions, using the same set of clinical specimens.

\section{Materials and Methods}

\section{Tissue samples}

All clinical samples used in this study were from FFPE blocks purchased from Molecular Response (San Diego, CA, USA) or Conversant Bio (Huntsville, AL, USA), and obtained with informed consent. All samples were reviewed by a certified pathologist to confirm the disease diagnosis and verify tumor content before IHC or GEP analysis. A total of 194 samples were first evaluated for disease diagnosis confirmation and then tested.

\section{Sample preparation, RNA extraction and GEP}

6 sections of $10 \mu \mathrm{m}$ each were cut from the tissue block per sample and de-paraffinized using xylene as described in the AllPrep DNA/ RNA handbook from QIAGEN (Valencia, CA, USA). Total RNA was isolated using the AllPrep DNA/RNA FFPE Kit (QIAGEN, catalog \#80234). Quality of RNA was assessed using an OD 260:280 ratio between 1.35 and 2.39, and the amount of DNA and RNA quantified using the NanoDrop ND8000 (Thermo Scientific, Waltham, MA USA) spectrophotometer.

\section{Hans-based IHC assay}

A Hans-based IHC method was used for testing at the IHC testing laboratory (Dako Laboratories, Carpinteria, CA, USA). This standardized Hans method was based on expression of CD10, BCL- 6 and MUM1/ IRF4 expression in FFPE diagnostic blocks, and depending on the sequential combination of expression that was observed, a sample was designated as GCB or non-GCB. The details of the method are described in the manuscript by Hans et al. [15]. Briefly, the samples were designated as GCB if they had either high CD10 or combination of low CD10, high BCL6 and low MUM1/IRF4 (Figure 1).

\section{Microarray gene expression profiling}

There are overlapping signature genes between each GEP platform as well as the genes corresponding to the proteins comprising the Hans-based IHC assay (Figure 2). Approximately $100 \mathrm{ng}$ of RNA from each sample (where the RNA quality was adequate and concentration was at least $6 \mathrm{ng} / \mu \mathrm{L}$ ) was amplified using the SensationPlus FFPE RNA Amplification Kit, and cDNA was labeled with biotin using the 3'IVT Labeling Kit (Affymetrix, Santa Clara, CA, USA; catalog \#901228). GEP was performed with the Affymetrix U133 Plus 2.0 Array according to the manufacturer's manual. Washing and staining processes were performed using the GeneChip hybridization wash and stain kit (Affymetrix, catalog \#900720). The resulting data were MAS5-normalized using the DNAcopy algorithm (www.bioconductor. org), and the expression measures for 200 probe sets were combined into a linear predictor score and DLBCL subtype was determined as previously reported [27]. 
Citation: Schaffer M, Chaturvedi S, Alvarez JD, Frans S, Aquino R, et al. (2018) Comparison of Immunohistochemistry Assay Results with Gene Expression Profiling Methods for Diffuse Large B-Cell Lymphoma Subtype Identification in Matched Patient Samples. J Mol Biomark Diagn 9: 386. doi: 10.4172/2155-9929.1000386

Page 3 of 8

\section{Lymphoma subtyping test assay for DLBCL classification}

Total RNA was extracted as described above, and it was ensured that samples had approximately $100 \mathrm{ng}$ of RNA before they were assessed using the research-use-only version of the LST, which uses a set of 5 housekeeping genes and 15 genes to differentiate between the $\mathrm{ABC}$ and GCB subtypes. The details of the assay methodology have been reported elsewhere [23].

\section{EdgeSeq COO assay for DLBCL classification}

Total RNA was extracted as described above and evaluated for DLBCL classification using the EdgeSeq COO platform, which is an NGS-based assay first reported in 2011 [26]. The list of genes has stayed constant, but revisions to the assay have been made to incorporate recent sequencing technology and to reduce the sample input.

\section{Sample classification for concordance}

In order to compare to the Hans-based IHC method, the sample subtype calls from the GEP methods were harmonized to either "GCB" or "non-GCB." All GCB calls remained GCB, and any "ABC" or "UNC" subtype calls from the microarray, LST and EdgeSeq COO platforms were converted to "non-GCB." Concordance between any pair of assays was calculated only using the total number of samples that could be called by both of those assays. Overall percent agreement and asymptotic $95 \%$ confidence intervals (CI) are presented. To determine the positive percent agreement (NPA) and negative percent agreement (NPA) (along with asymptotic 95\% CI), the microarray-based or, more generally, the GEP-based subtyping methods served as nonstandard references in each comparison.

\section{Results}

Of the 194 samples first evaluated for disease diagnosis confirmation, 137 were confirmed as DLBCL and had sufficient material available for both IHC and at least 1 GEP-based method. Among these, an almost equivalent number were GCB and non-GCB according to the Hansbased IHC assay: $48.9 \%$ (67/137) and 51.1\% (70/137) of the samples, respectively. The median age of the patients sampled was 69 years, and $45.0 \%$ were male. $56.3 \%$ of patients received prior therapy.

Concordance of the Hans-based IHC method versus the microarray assay

All 137 samples evaluated by the Hans-based IHC method were compared with the DNA microarray-based GEP method. The microarray algorithm classified 36 of the 137 samples as ABC, 18 as UNC and 83 as GCB (Figure 3A). The agreement between the 2 methods was generally better for non-GCB than for GCB calls, with only $1 / 36 \mathrm{ABC}$ (by microarray) sample being called GCB by IHC. Of the 54 samples called non-GCB by microarray GEP, 48 were also called non-GCB by
Hans-based IHC, leading to a PPA between these 2 methods in calling non-GCB (using the GEP method as nonstandard reference) of 48 of 54, 88.9\% (95\% CI, 80.5-97.3). Interestingly, the NPA in the assignment of GCB was lower $(61 / 83,73.5 \%$; $95 \%$ CI, 64.0-83.0), suggesting that the larger variance between the methods was aligned with GCB calls. The overall concordance for these 2 methods in identifying either subtype was 109 of 137, 79.6\% (95\% CI, 72.8-86.3) (Figure 3A).

\section{Concordance of the Hans-based IHC method versus LST}

A total of 125 samples were classified by both the Hans-based IHC assay and the LST. Of these, the LST classified 41 samples as $\mathrm{ABC}, 13$ as UNC and 71 as GCB. Of the 54 samples that were called non-GCB using LST, 47 were also classified as non-GCB using the Hans-based IHC assay, giving a PPA of 47 of 54, 87.0\% (95\% CI, 78.196.0). For GCB, 53 of the 71 samples called GCB using the LST were also called GCB by the Hans-based IHC assay, leading to an NPA of 53 of $71,74.6 \%$ (95\% CI, 64.5-84.8). Overall concordance for the Hans-based IHC assay with the LST method was nearly identical $(100 / 125,80.0 \%$; $95 \% \mathrm{CI}, 73.0-87.0)$ to that with the microarray-GEP method (Figure 3B).

\section{Concordance of Hans-based IHC method versus EdgeSeq COO assay}

The EdgeSeq COO assay uses a completely different technology (NGS) compared with other GEP methods to quantitate the RNA expression levels, and we compared this to the Hans-based IHC method as well as the other GEP methods. Only a total of 64 samples were available for testing by the EdgeSeq $\mathrm{COO}$ assay, which were classified as $28 \mathrm{ABC}, 4 \mathrm{UNC}$ and $32 \mathrm{GCB}$. Of these, 32 were called GCB and 32 were called non-GCB by the Hans-based IHC assay. Within this smaller subset, for each of the GCB and non-GCB groups, 25 of 32 calls were aligned, demonstrating an overall concordance of 50 of $64,78.1 \%(95 \%$ CI, 68.0-88.3) (Figure 3C).

\section{Concordance between gene expression profiling assays}

It was of interest to compare the newer GEP approaches with that of the traditional microarray-based assay because, unlike the IHC methods, they all measure RNA expression on completely different technology platforms. Similar to the microarray assay, the LST and EdgeSeq assays were developed to have 3 categories (ABC, GCB and UNC). So, to compare the concordance between these methods and the IHC results, these were assigned to GCB and non-GCB categories as described in the Materials and Methods. During their development, the LST and EdgeSeq $\mathrm{COO}$ assays were calibrated to the published microarraybased GEP method. As expected, the LST method showed a high level of overall concordance with microarray at $93.7 \%$ (118/126), including correct assignment of all $35 \mathrm{ABC}$ samples identified by microarray. The PPA was $94.3 \%$ (50/53) and the NPA was $93.2 \%$ (68/73) using the

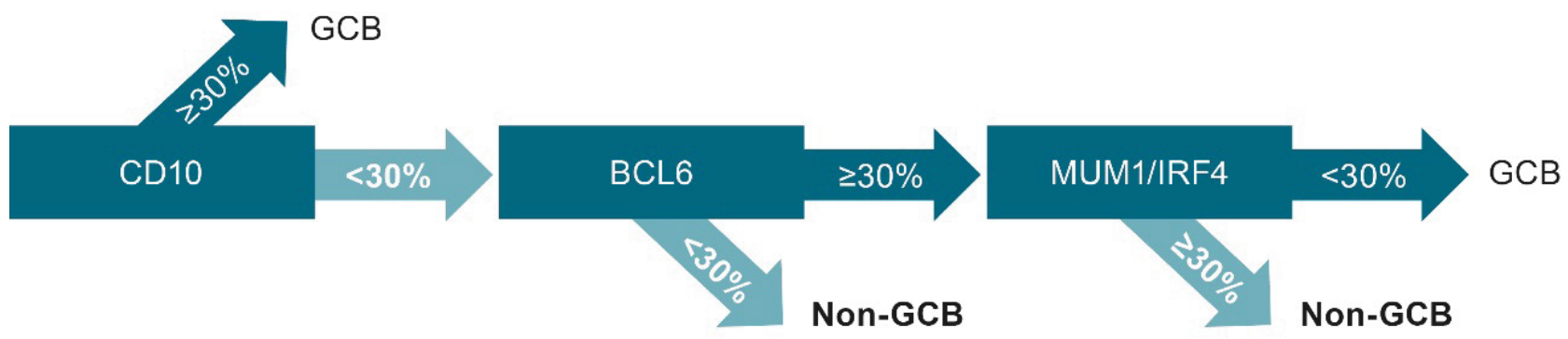

Figure 1: Hans-based immunohistochemistry algorithm for identifying GCB subtype DLBCL. DLBCL: Diffuse Large B-Cell Lymphoma; GCB: Germinal Center B-Cell-Like. 


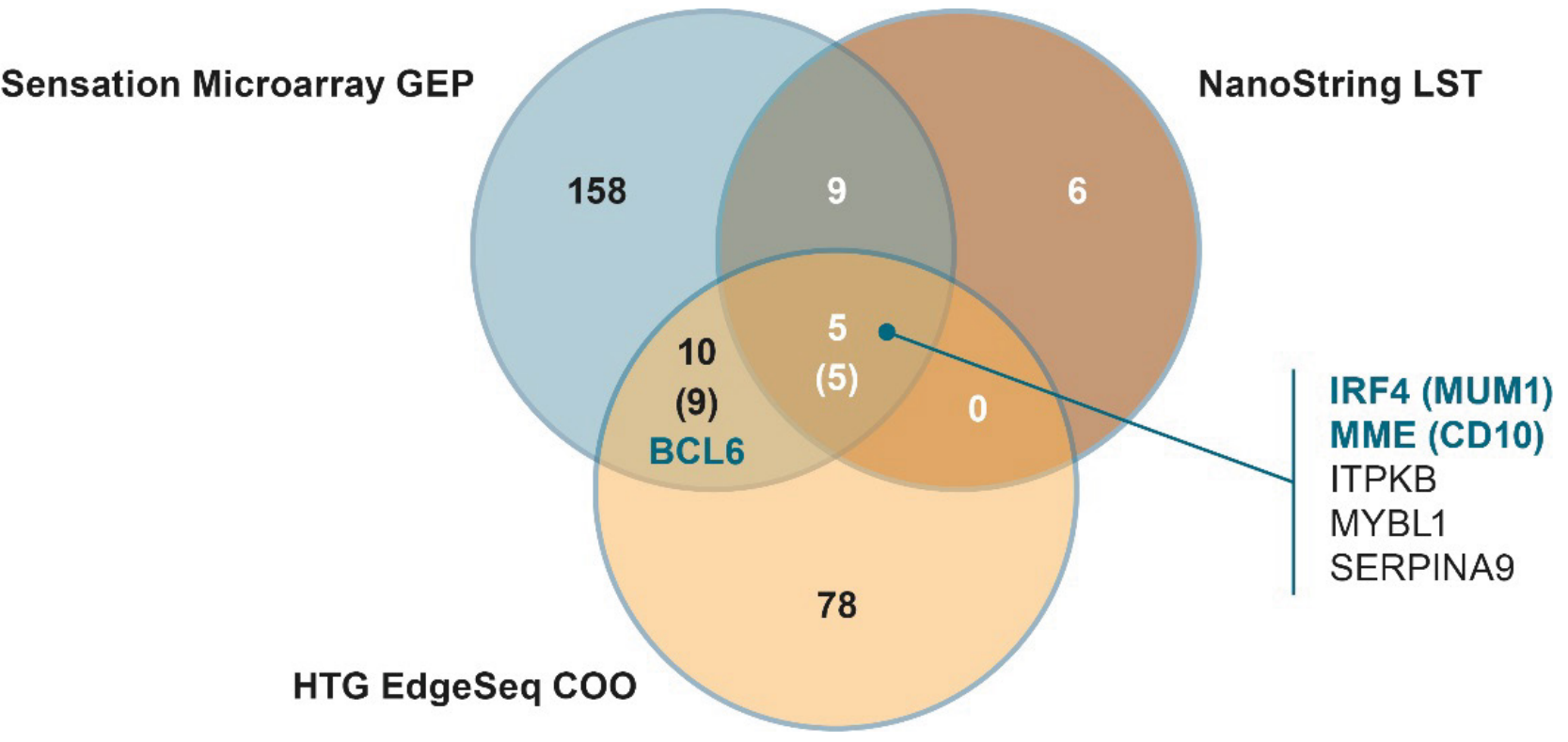

Figure 2: GEP signature genes. Genes encoding the Hans-based IHC markers are indicated as bolded text. The overlap of the 14 COO markers from the EdgeSeq COO method are indicated in parentheses. COO: Cell of Origin; GEP: Gene Expression Profiling; IHC: Immunohistochemistry; LST: Lymphoma Subtyping Test.

microarray GEP method as the reference. Interestingly, the LST assay classified a total of 42 samples as $\mathrm{ABC}$, which also included $2 \mathrm{GCB}$ and 5 UNC samples as defined by microarray, leading to a concordance of $83.3 \%$ in identifying ABC between these 2 GEP methods. The EdgeSeq $\mathrm{COO}$ assay showed good overall concordance with the microarray method $(56 / 64,87.5 \%)$ with a PPA of $92.9 \%(26 / 28)$ and an NPA of 83.3\% (30/36), demonstrating consistency between GCB and non-GCB assignments for the tested, method-aligned GEP approaches.

\section{Concordance of all assays across each sample}

Overall, the distribution of $\mathrm{ABC}, \mathrm{UNC}$ and non-GCB was similar by the GEP assays, but with some notable trends (Figure 4A). It is interesting that the EdgeSeq assay reports fewer UNC calls (6.3\%) than the other 2 GEP methods $(13.1 \%$ and $10.4 \%$ for microarray and LST, respectively), with a concurrent increase in the percentage of $\mathrm{ABC}(43.8 \%$ by EdgeSeq vs $26.3 \%$ and $32.8 \%$ for microarray and LST, respectively). Figure $4 \mathrm{~B}$ shows the collection of all samples used in this study and displays the assay identification for each. In the vertical extremes of the figure, it is clear that the calls for each sample are highly concordant. However, in the region surrounding the UNC calls generated by the microarray method, the concordance becomes less evident. Nonetheless, the comparison of different assays shows that overall, the concordance and the PPA for non-GCB calls between the Hans-based IHC assay and the 3 GEP assays are similar (concordance of $79.6 \%, 80.0 \%$ and $78.1 \%$ and PPA of $88.9 \%, 87 \%$ and $78.1 \%$ for the microarray-based, LST and EdgeSeq assays, respectively) and comparable to that between the GEP assays themselves (Figure 4C).

\section{Discussion}

With the progressive advancement of biomarkers over the past decade, there has been a greater appreciation for heterogeneity of diseases. In our study, $26.3 \%$ to $43.7 \%$ of the DLBCL samples were classified as $\mathrm{ABC}, 50.0 \%$ to $60.6 \%$ as GCB and $6.3 \%$ to $13.1 \%$ as UNC. The distribution of these main subtypes is generally consistent with previous reports $[4,28]$.
COO has shown to be a prognostic factor in DLBCL $[7,9,29]$. In a retrospective analysis of de novo cases of DLBCL using the Hans IHC approach as surrogate for GEP-based COO molecular classification, the non-GCB subtype was associated with a higher risk of progression and reduced survival when conventional R-CHOP regimens are used [7]. In another study, the Hans method has been used in the clinical trial LNH $03-2 B$ to show that low to intermediate risk (per the International Prognostic Index) non-GCB DLBCL patients had a survival advantage when treated with dose-intensive R-ACVBP (rituximab, doxorubicin, cyclophosphamide, vindesine, bleomycin and prednisone) compared with standard R-CHOP therapy [30]. Chronic active B-cell receptor signaling has been demonstrated in the ABC subtype of DLBCL [31], which appears to be particularly responsive to BTK inhibitors [32], suggesting that future studies may be able to identify rational targets for drug intervention in DLBCL subtypes identified using COO. However, the prognostic value of $\mathrm{COO}$ has not been confirmed with other classifications or treatment regimens. For example, in a retrospective study using samples from the RICOVER-60 and R-Mega-CHOEP trials in patients with DLBCL, where COO subgroups were identified via Lymph2Cx and IHC assays, COO profiling alone failed to identify prognostic subgroups, although MYC/BCL2 double expression was predictive of poor survival [33]. Furthermore, some treatment regimens may be effective across DLBCL subtypes. For example, in a phase Ib study evaluating ibrutinib plus R-CHOP in treatment-naïve CD20-positive B-cell NHL, all patients $(n=23)$ with DLBCL had a complete or partial response for an overall response rate of $95 \%$. Interestingly, of the patients who were subtyped by Hans-based IHC, 2 (100\%) with non-GCB subtype but only 5 of 7 (71.4\%) with GCB subtype had a complete response [34].

The field of DLBCL has been inundated with several assays, each attempting to differentiate between the GCB, ABC, UNC or the nonGCB cohorts. In our study, a single standardized Hans-based IHC assay was used as a base or reference method against which select GEP methods were compared. While each assay has its own limitations, the availability of a standardized IHC kit makes it an attractive assay that can serve as a rapid, cost-effective and accessible platform in most clinical centers. 
Citation: Schaffer M, Chaturvedi S, Alvarez JD, Frans S, Aquino R, et al. (2018) Comparison of Immunohistochemistry Assay Results with Gene Expression Profiling Methods for Diffuse Large B-Cell Lymphoma Subtype Identification in Matched Patient Samples. J Mol Biomark Diagn 9: 386. doi: 10.4172/2155-9929.1000386

\begin{tabular}{|c|c|c|c|c|c|}
\cline { 3 - 6 } \multicolumn{2}{c|}{} & \multicolumn{4}{c|}{ Microarray } \\
\cline { 2 - 6 } \multicolumn{2}{c|}{} & ABC & GCB & UNC & Sum \\
\hline $\begin{array}{c}\text { Hans-based } \\
\text { IHC }\end{array}$ & Non-GCB & 35 & 22 & 13 & 70 \\
\cline { 2 - 6 } & GCB & 1 & 61 & 5 & 67 \\
\hline & Sum & 36 & 83 & 18 & 137 \\
\cline { 2 - 6 }
\end{tabular}

Overall percent agreement $(95 \% \mathrm{Cl})=109 / 137,79.6 \%(72.8-86.3)$

Positive percent agreement (non-GCB, 95\% Cl) $=48 / 54,88.9 \%(80.5-97.3)$

Negative percent agreement $(\mathrm{GCB}, 95 \% \mathrm{Cl})=61 / 83,73.5 \%(64.0-83.0)$

B

\begin{tabular}{|c|c|c|c|c|c|}
\hline & \multicolumn{4}{|c|}{ LST } \\
\hline & & $A B C$ & GCB & UNC & Sum \\
\hline \multirow{3}{*}{$\begin{array}{c}\text { Hans-based } \\
\text { IHC }\end{array}$} & Non-GCB & 37 & 18 & 10 & 65 \\
\hline & GCB & 4 & 53 & 3 & 60 \\
\hline & Sum & 41 & 71 & 13 & 125 \\
\hline
\end{tabular}

Overall percent agreement $(95 \% \mathrm{Cl})=100 / 125,80.0 \%(73.0-87.0)$

Positive percent agreement (non-GCB, 95\% Cl) $=47 / 54,87.0 \%(78.1-96.0)$

Negative percent agreement $(\mathrm{GCB}, 95 \% \mathrm{Cl})=53 / 71,74.6 \%(64.5-84.8)$

\begin{tabular}{|c|c|c|c|c|c|}
\cline { 3 - 6 } \multicolumn{2}{c|}{} & \multicolumn{4}{c|}{ EdgeSeq COO } \\
\cline { 2 - 6 } \multicolumn{2}{c|}{} & ABC & GCB & UNC & Sum \\
\hline $\begin{array}{c}\text { Hans-based } \\
\text { IHC }\end{array}$ & Non-GCB & 24 & 7 & 1 & 32 \\
\cline { 2 - 6 } & GCB & 4 & 25 & 3 & 32 \\
\hline & Sum & 28 & 32 & 4 & 64 \\
\cline { 2 - 6 }
\end{tabular}

Overall percent agreement $(95 \% \mathrm{Cl})=50 / 64,78.1 \%(68.0-88.3)$

Positive percent agreement (non-GCB, 95\% Cl) $=25 / 32$, 78.1\% (63.8-92.4)

Negative percent agreement $(\mathrm{GCB}, 95 \% \mathrm{Cl})=25 / 32,78.1 \%(63.8-92.4)$

Figure 3: Overall concordance between methods of identifying subtypes of DLBCL. A) Hans-based IHC versus microarray assay. B) Hans-based IHC versus LST. C) Hans-based IHC versus EdgeSeq COO. ABC: Activated B-Cell-Like; Cl: Confidence Interval; DLBCL: Diffuse Large B-Cell Lymphoma; GCB: Germinal Center B-Cell-Like; IHC: Immunohistochemistry; LST: Lymphoma Subtyping Test; UNC: Unclassified. 
Citation: Schaffer M, Chaturvedi S, Alvarez JD, Frans S, Aquino R, et al. (2018) Comparison of Immunohistochemistry Assay Results with Gene Expression Profiling Methods for Diffuse Large B-Cell Lymphoma Subtype Identification in Matched Patient Samples. J Mol Biomark Diagn 9: 386. doi: 10.4172/2155-9929.1000386

A

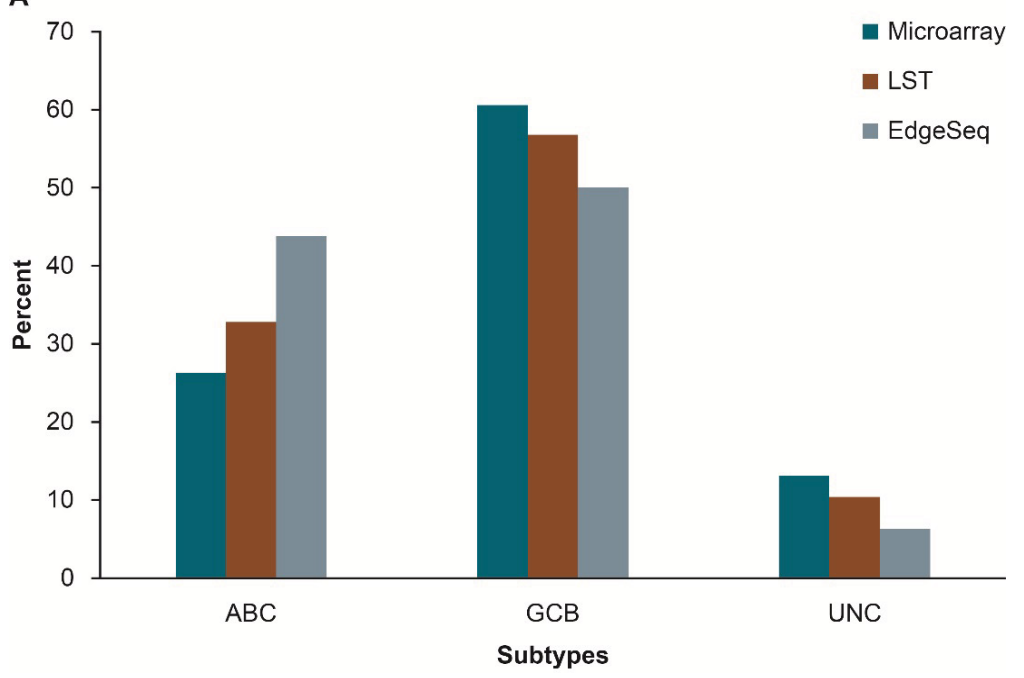

C
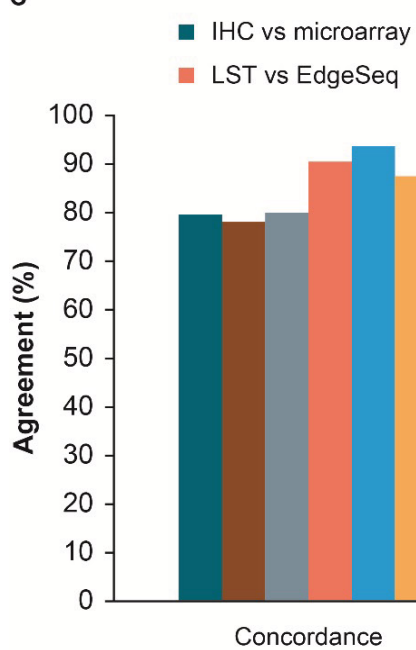

- IHC vs EdgeSeq

- LST vs microarray

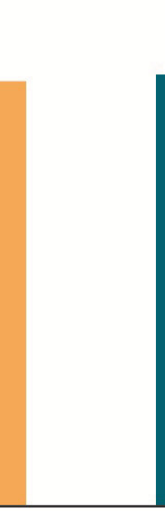

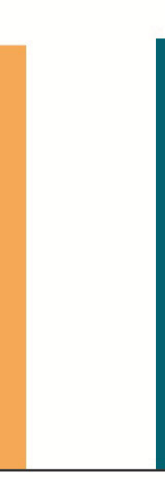

(1)

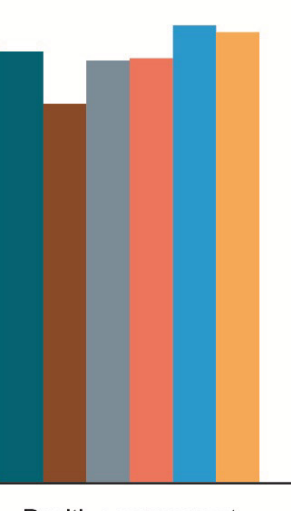

Positive agreement
B

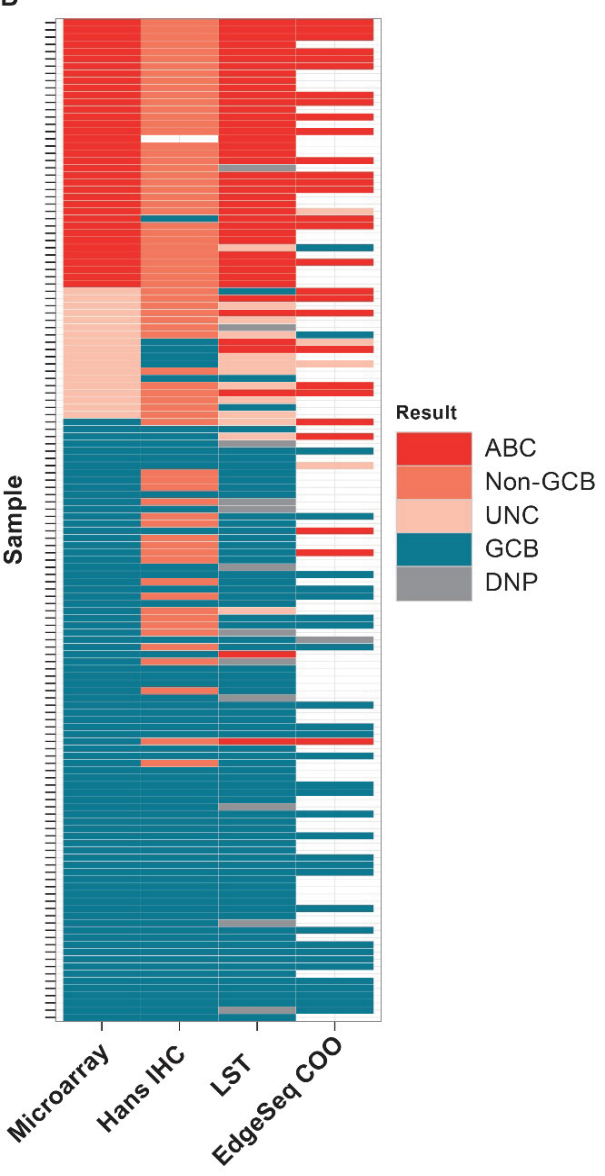

IHC vs LST

EdgeSeq vs microarray

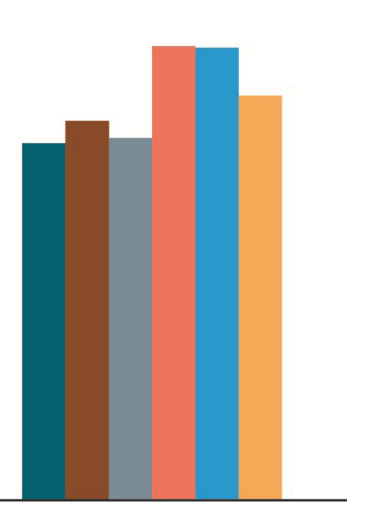

Negative agreement

Figure 4: Distribution of DLBCL subtypes and concordance by assays. A) Distribution of DLBCL subtype by GEP. B) Comparison of assay concordance between samples. C) Concordance and percent agreement by assays. ABC: Activated B-Cell-Like; COO: Cell of Origin; DLBCL: Diffuse Large B-Cell Lymphoma; DNP: Did Not Pass; GCB: Germinal Center B-Cell-Like; GEP: Gene Expression Profiling; IHC: Immunohistochemistry; LST: Lymphoma Subtyping Test; UNC: Unclassified.

Several IHC-based assays have been evaluated in the past, and a very detailed analysis shows that GCB and non-GCB subtypes of DLBCL can be accurately predicted with the Hans assay using a panel of 3 IHC stains: CD10, BCL6 and MUM1 [15]. The antibodies selected for each of these stains recognize molecules whose mRNA expression was highly associated with GCB and non-GCB groups in the microarray studies [3,4]. The Hans algorithm is an acceptable algorithm to differentiate GCB from non-GCB tumor cells according to the updated World Health Organization classification; in addition, a previous study found a Hans-based IHC method to have $86 \%$ concordance 
with microarray GEP $[19,21,35]$. In our study, concordance between the Hans-based IHC assay, the microarray-based LST assay and the EdgeSeq assay was $79.6 \%, 80.0 \%$ and $78.2 \%$, respectively, consistent with previous reported concordance $[17,19]$. Classifications by the 3 commercially available GEPs were also aligned, with concordances ranging from $87.5 \%$ to $93.7 \%$. Other studies suggest that $\geq 70 \%$ to $80 \%$ concordance of other methods with GEP is sufficient to differentiate patients and choose appropriate treatment modalities [17,19]; however, GEP methods generally show a greater prognostic difference between subtypes, demonstrating the consistency with the original $\mathrm{COO}$ hypothesis [36].

GEP using traditional microarrays has been utilized extensively in the recent past to observe differences between the subtypes of DLBCL. While it is a suitable tool for research in a laboratory setting or as a part of exploratory analysis, this method requires RNA isolated preferably from fresh tissue, which can pose a challenge in a clinical setting, and also lacks a common, easily available commercial assay for routine clinical practice. Additionally, there is no consistently applied microarray gene set and corresponding algorithm reported in the literature, despite many subtyping publications over many years. And while the development of the new GEP-based methods has attempted to formalize these previously reported approaches to a consistent assay, there is no unique, publicly available sample set used as a standardized approach to defining different subtypes of DLBCL by the various technologies available today. Therefore, in this study, a large set of commercially purchased and well-defined DLBCL samples was used to provide a single matched set that could provide materials for both IHC and a variety of GEP-based assays. Using these samples, which were classified by the accepted standard, well-published microarray-based $\mathrm{COO}$ assay, a platform was created for comparison of a number of other IHC and GEP-based methods. These may soon become commercially available and could be used in clinical settings to guide optimal choice of treatment, a consideration that is becoming ever more important with the increasing number of targeted COO-based DLBCL therapies currently in clinical development.

The LST provides a close analogue of the microarray-based GEP method, as it is also an RNA-based expression analysis method and uses the same reference sample set and classification for input. However, the method developed was more robust and quantitative, using a platform that was approved by the US Food and Drug Administration and based on using only 20 genes for expression analysis on FFPE tissue. 5 of these genes were "housekeeping" genes for normalization, and the 15 other analyte genes were part of the gene set established by Lenz et al. to accurately predict the COO assignment [23]. Because the GEP platform was the original way of defining the DLBCL subtypes based on COO, it was important to establish that a GEP-based COO method was highly concordant with the Hans-based IHC method, so patients' diseases could be accurately identified regardless of the platform available at their particular center and chosen by their physician. The results, with $80 \%$ overall concordance and $87 \%$ PPA in identifying nonGCB patients, show that this is indeed the case.

The more recent EdgeSeq COO method, on the other hand, provides a completely different NGS-based platform that has lower sample requirements, but is also in essence an RNA expression-based assay. This method also shows good concordance with the Hans-based IHC method, although it is notable that the algorithm appears to reduce the percentage of UNC calls compared with the other GEP methods, while increasing the percentage of $\mathrm{ABC}$ calls. The prognostic significance of this variance remains to be determined, but it is worth noting that both of these subtypes are included in the non-GCB call by IHC.
It is all the more remarkable that a standardized IHC-based assay, using a very different source material and using a binary (as opposed to a ternary) method requiring human evaluation, can provide a high concordance of $80 \%$ between IHC and all of the evaluated GEP methods. One difference is that GEP-based methods allow identification specifically of the ABC subset versus only non-GCB identification produced by the Hans-based IHC method. However, the concordance between the GEP-based methods for identifying $\mathrm{ABC}$ patients is also similar, at $83.3 \%$ (26.3\% by microarray, $32.8 \%$ by LST and $43.8 \%$ by EdgeSeq), although the PPA for non-GCB is higher at $87.5 \%$ to $94.3 \%$. Because GEP methods use a continuous score based on the expression levels of the genes in the algorithm, it becomes difficult to clearly assign the boundaries of the unclassified subgroup, leading to the variance in the $\mathrm{ABC}$ versus UNC calls between these methods noted above. Thus, overall within a margin of error, it becomes apparent that the results here show that these methods are concordant and can provide acceptable, alternate ways to subtype DLBCL to guide appropriate therapy choices.

This analysis has inherent limitations. Specifically, the specimens used were from a commercial source with no clinical follow-up. Therefore, characteristics of the patients and the ultimate results of their therapy are not available.

\section{Conclusion}

In summary, herein we have described a robust, optimized Hansbased IHC assay using a standard set of reagents and an optimized algorithm in a central laboratory that offers a relatively inexpensive and accessible platform for COO subtyping in DLBCL. With clinical validation from an ongoing phase III study evaluating ibrutinib efficacy and safety in non-GCB patients, this platform may offer an excellent tool for the identification of DLBCL subtypes, and thus potentially in the selection of optimal therapy. We have also shown that there is a high concordance of this IHC assay with GEP-based methods such as microarray, LST and EdgeSeq COO, and also between the various GEP-based methods among themselves. Given these results, it is clear that with some further development and validation, a number of robust, standardized and equivalent methods may become available in the marketplace, increasing the choice and availability to ensure that DLBCL patients in any location worldwide can be rapidly and accurately classified to receive optimal therapy. Several ongoing studies are considering $\mathrm{COO}$ subtypes in the evaluation of treatment efficacy in DLBCL; the results of these studies may provide further insight into appropriate treatments for patients with different DLBCL subtypes [22,37-41].

\section{Acknowledgments}

This study was funded by Janssen Research \& Development. Funders were involved in the study design, data collection, data analysis and interpretation. Lead investigators had full access to the data and analyses for compilation of this report. Writing assistance was provided by Min Yu of PAREXEL and was funded by Janssen Global Services, LLC.

\section{Conflicts of interest}

MS, SC, JA, SF and RA are employees of Janssen. JP and BH have nothing to disclose. MW is an employee and has equity ownership at Janssen. SB is an employee and has equity ownership at Janssen and has equity ownership at Pharmacyclics.

\section{References}

1. Siegel RL, Miller KD, Jemal A (2016) Cancer statistics, 2016. CA Cancer J Clin 66: 7-30.

2. Dunleavy K, Pittaluga S, Maeda LS, Advani R, Chen CC, et al. (2013) Doseadjusted EPOCH-rituximab therapy in primary mediastinal B-cell lymphoma. N Engl J Med 368: 1408-1416.

3. Alizadeh AA, Eisen MB, Davis RE, Ma C, Lossos IS, et al. (2000) Distinct types of diffuse large B-cell lymphoma identified by gene expression profiling. Nature 403: 503-511. 
Citation: Schaffer M, Chaturvedi S, Alvarez JD, Frans S, Aquino R, et al. (2018) Comparison of Immunohistochemistry Assay Results with Gene Expression Profiling Methods for Diffuse Large B-Cell Lymphoma Subtype Identification in Matched Patient Samples. J Mol Biomark Diagn 9: 386. doi: 10.4172/2155-9929.1000386

4. Rosenwald A, Wright G, Chan WC, Connors JM, Campo E, et al. (2002) The use of molecular profiling to predict survival after chemotherapy for diffuse large-B-cell lymphoma. N Engl J Med 346: 1937-1947.

5. Lenz G, Wright G, Dave SS, Xiao W, Powell J, et al. (2008) Stromal gene signatures in large-B-cell lymphomas. N Engl J Med 359: 2313-2323.

6. Rosenwald A, Wright G, Leroy K, Yu X, Gaulard P, et al. (2003) Molecular diagnosis of primary mediastinal $\mathrm{B}$ cell lymphoma identifies a clinically favorable subgroup of diffuse large B cell lymphoma related to Hodgkin lymphoma. J Exp Med 198: 851-862.

7. Batlle-Lopez A, Gonzalez de Villambrosia S, Francisco M, Malatxeberria S Saez A, et al. (2016) Stratifying diffuse large B-cell lymphoma patients treated with chemoimmunotherapy: GCB/non-GCB by immunohistochemistry is still a robust and feasible marker. Oncotarget 7: 18036-180349.

8. Ozbalak M, Ar MC, Tuzuner N, Salihoglu A, Eskazan AE, et al. (2013) Detailed analysis of diffuse large $B$ cell lymphoma patients: a single-center, retrospective study. ISRN Hematol 2013: 908191.

9. Rovira J, Karube K, Valera A, Colomer D, Enjuanes A et al. (2016) MYD88 L265P mutations, but no other variants, identify a subpopulation of DLBCL patients of activated B-cell origin, extranodal involvement, and poor outcome. Clin Cancer Res 22: 2755-2764.

10. Bachy E, Salles G (2015) Treatment approach to newly diagnosed diffuse large B-cell lymphoma. Semin Hematol 52: 107-118.

11. Wilson WH, Young RM, Schmitz R, Yang Y, Pittaluga S, et al. (2015) Targeting $\mathrm{B}$ cell receptor signaling with ibrutinib in diffuse large $\mathrm{B}$ cell lymphoma. Nat Med 21: 922-926.

12. Nowakowski GS, LaPlant B, Macon WR, Reeder CB, Foran JM, et al. (2015) Lenalidomide combined with $\mathrm{R}-\mathrm{CHOP}$ overcomes negative prognostic impact of non-germinal center B-cell phenotype in newly diagnosed diffuse large B-cell lymphoma: a phase II study. J Clin Oncol 33: 251-257.

13. Poulsen CB, Borup R, Nielsen FC, Borregaard N, Hansen M, et al. (2005) Microarray-based classification of diffuse large B-cell lymphoma. Eur J Haematol 74: 453-465

14. Coutinho R, Clear AJ, Owen A, Wilson A, Matthews J, et al. (2013) Poor concordance among nine immunohistochemistry classifiers of cell-of-origin for diffuse large B-cell lymphoma: implications for therapeutic strategies. Clin Cancer Res 19: 6686-6695

15. Hans CP, Weisenburger DD, Greiner TC, Gascoyne RD, Delabie J, et al. (2004) Confirmation of the molecular classification of diffuse large B-cell lymphoma by immunohistochemistry using a tissue microarray. Blood 103: 275-282.

16. Gang $A O$, Pedersen $M O$, Knudsen $H$, Lauritzen AF, Pedersen $M$, et al (2015) Cell of origin predicts outcome to treatment with etoposide-containing chemotherapy in young patients with high-risk diffuse large B-cell lymphoma. Leuk Lymphoma 56: 2039-2046.

17. Friedberg JW (2015) Using the pathology report in initial treatment decisions for diffuse large B-cell lymphoma: time for a precision medicine approach. Hematology Am Soc Hematol Educ Program 2015: 618-624.

18. Hwang HS, Park CS, Yoon DH, Suh C, Huh J (2014) High concordance of gene expression profiling-correlated immunohistochemistry algorithms in diffuse large B-cell lymphoma, not otherwise specified. Am J Surg Pathol 38: 1046-1057.

19. Meyer PN, Fu K, Greiner TC, Smith LM, Delabie J, et al. (2011) Immunohistochemical methods for predicting cell of origin and survival in patients with diffuse large B-cell lymphoma treated with rituximab. J Clin Onco 29: 200-207

20. Read JA, Koff JL, Nastoupil LJ, Williams JN, Cohen JB, et al. (2014) Evaluating cell-of-origin subtype methods for predicting diffuse large B-cell lymphoma survival: a meta-analysis of gene expression profiling and immunohistochemistry algorithms. Clin Lymphoma Myeloma Leuk 14: 460-467.

21. Swerdlow SH, Campo E, Pileri SA, Harris NL, Stein H, et al. (2016) The 2016 revision of the World Health Organization classification of lymphoid neoplasms. Blood 127: 2375-2390.

22. Clinicaltrials.gov. NCT01855750. https://clinicaltrials.gov/ct2/show/NCT01855750. Last update: Jul 6, 2017. Accessed Oct 6, 2017.
23. Scott DW, Wright GW, Williams PM, Lih CJ, Walsh W, et al. (2014) Determining cell-of-origin subtypes of diffuse large B-cell lymphoma using gene expression in formalin-fixed paraffin-embedded tissue. Blood 123: 1214-1217.

24. Scott DW, Mottok A, Ennishi D, Wright GW, Farinha P, et al. (2015) Prognostic significance of diffuse large B-cell lymphoma cell of origin determined by digital gene expression in formalin-fixed paraffin-embedded tissue biopsies. J Clin Oncol 33: 2848-2856.

25. Clinicaltrials.gov. NCT02285062. https://clinicaltrials.gov/ct2/show/NCT02285062. Last update: Sep 29, 2017. Accessed Oct 6, 2017.

26. Rimsza LM, Wright G, Schwartz M, Chan WC, Jaffe ES, et al. (2011) Accurate classification of diffuse large B-cell lymphoma into germinal center and activated B-cell subtypes using a nuclease protection assay on formalin-fixed, paraffin-embedded tissues. Clin Cancer Res 17: 3727-3732.

27. Lenz G, Wright GW, Emre NC, Kohlhammer H, Dave SS, et al. (2008) Molecular subtypes of diffuse large B-cell lymphoma arise by distinct genetic pathways Proc Natl Acad Sci U S A 105: 13520-13525.

28. Mareschal S, Lanic H, Ruminy P, Bastard C, Tilly H, et al. (2011) The proportion of activated B-cell like subtype among de novo diffuse large B-cell lymphoma increases with age. Haematologica 96: 1888-1890.

29. Lu TX, Miao Y, Wu JZ, Gong QX, Liang JH, et al. (2016) The distinct clinica features and prognosis of the CD10(+)MUM1(+) and CD10(-)Bcl6(-)MUM1(-) diffuse large B-cell lymphoma. Sci Rep 6: 20465

30. Molina TJ, Canioni D, Copie-Bergman C, Recher C, Briere J, et al. (2014) Young patients with non-germinal center B-cell-like diffuse large B-cell lymphoma benefit from intensified chemotherapy with ACVBP plus rituximab compared with $\mathrm{CHOP}$ plus rituximab: analysis of data from the Groupe d'Etudes des Lymphomes de l'Adulte/lymphoma study association phase III trial LNH 03-2B. J Clin Oncol 32: 3996-4003.

31. Davis RE, Ngo VN, Lenz G, Tolar P, Young RM, et al. (2010) Chronic active B-cell-receptor signalling in diffuse large B-cell lymphoma. Nature 463: 88-92.

32. Staudt LM, Dunleavy K, Buggy JJ, Hedrick E, Lucas N, et al. (2011) The Bruton's Tyrosine Kinase (Btk) inhibitor PCl-32765 modulates chronic active $B C R$ signaling and induces tumor regression in relapsed/refractory $A B C$ DLBCL. Blood 118: abstract 2716.

33. Staiger AM, Ziepert M, Horn H, Scott DW, Barth TFE, et al. (2017) Clinical impact of the cell-of-origin classification and the MYC/ BCL2 dual expresse status in diffuse large B-cell lymphoma treated within prospective clinical trials of the German High-Grade Non-Hodgkin's Lymphoma Study Group. J Clin Oncol 35: 2515-2526.

34. Younes A, Thieblemont C, Morschhauser F, Flinn I, Friedberg JW, et al. (2014 Combination of ibrutinib with rituximab, cyclophosphamide, doxorubicin vincristine, and prednisone (R-CHOP) for treatment-naive patients with CD20 positive B-cell non-Hodgkin lymphoma: a non-randomised, phase $1 \mathrm{~b}$ study Lancet Oncol 15: 1019-26.

35. Campo E, Swerdlow SH, Harris NL, Pileri S, Stein H, et al. (2011) The 2008 WHO classification of lymphoid neoplasms and beyond: evolving concepts and practical applications. Blood 117: 5019-5032. (http://www.ncbi.nlm.nih.gov/ pubmed/21300984)

36. Gutierrez-Garcia G, Cardesa-Salzmann T, Climent F, Gonzalez-Barca E Mercadal S, et al. (2011) Gene-expression profiling and not immunophenotypic algorithms predicts prognosis in patients with diffuse large B-cell lymphoma treated with immunochemotherapy. Blood 117: 4836-4843.

37. Clinicaltrials.gov. NCT01563861. https://clinicaltrials.gov/ct2/show/NCT01563861. Last update: Dec 19, 2016. Accessed Oct 6, 2017.

38. Clinicaltrials.gov. NCT01856192. https://clinicaltrials.gov/ct2/show/NCT01856192. Last update: Oct 6, 2017. Accessed Oct 6, 2017.

39. Clinicaltrials.gov. NCT03003520. https://clinicaltrials.gov/ct2/show/results/ NCT03003520. Last update: Oct 3, 2017. Accessed Oct 6, 2017.

40. Clinicaltrials.gov. NCT02391116. https://clinicaltrials.gov/ct2/show/NCT02391116. Last update: Aug 31, 2017. Accessed Oct 6, 2017.

41. Clinicaltrials.gov. NCT01325701. https://clinicaltrials.gov/ct2/show/NCT01325701. Last update: Mar 31, 2017. Accessed Oct 6, 2017. 